\title{
Cannabis treatment outcomes among legally coerced and non-coerced adults Jan Copeland ${ }^{* \dagger 1}$ and Jane C Maxwell ${ }^{\dagger 2}$
}

\author{
Address: ${ }^{1}$ National Drug and Alcohol Research Centre, University of New South Wales, Sydney, 2052, Australia and ${ }^{2}$ Addiction Research Institute, \\ Center for Social Work Research, University of Texas at Austin, USA \\ Email: Jan Copeland* - J.Copeland@unsw.edu.au; Jane C Maxwell - jcmaxwell@mail.utexas.edu \\ * Corresponding author †Equal contributors
}

Published: 14 June 2007

BMC Public Health 2007, 7:1 II doi:10.1 I86/147|-2458-7-III
Received: 28 January 2007

Accepted: 14 June 2007

This article is available from: http://www.biomedcentral.com/I47I-2458/7/III

(c) 2007 Copeland and Maxwell; licensee BioMed Central Ltd.

This is an Open Access article distributed under the terms of the Creative Commons Attribution License (http://creativecommons.org/licenses/by/2.0), which permits unrestricted use, distribution, and reproduction in any medium, provided the original work is properly cited.

\begin{abstract}
Background: Treatment seeking for cannabis dependence in general, and particularly the number of criminal justice referrals to cannabis treatment, has increased over the past decade. This study aims to compare the characteristics, psychosocial functioning and treatment outcome of those legally coerced into cannabis treatment compared to those entering treatment without legal coercion.
\end{abstract}

Methods: This study is a retrospective audit of the administrative clinical records of 27,198 adults presenting to public Texas treatment programs with cannabis as their primary drug problem between 2000 and 2005.

Results: Of the $69 \%$ legally coerced into treatment, there was less psychological distress and greater likelihood of having completed treatment compared with non-coerced clients. Participants who were legally coerced into treatment were also more likely to have received less intensive forms of treatment and to have not used cannabis in the month prior to 90-day post-treatment follow-up.

Conclusion: More public health information is needed on cannabis dependence and increased availability of subsidised early and brief interventions in a variety of primary health care settings would reduce the late presentations of the more severely impaired voluntary clients. The limitations of this dataset are discussed.

\section{Background}

Substance use and related problems have presented an increasing challenge for most countries over the past few decades [1]. Cannabis is the most commonly used of the illicit drugs internationally [2]. A substantial proportion of cannabis users develop cannabis-related problems, including abuse and dependence $[3,4]$. Recent analyses comparing the past year prevalence of cannabis use disorder in large US epidemiological studies between 1991 and 2002 found significant increases from $1.2 \%$ to $1.5 \%$ with disproportionate increases in African-American and Hispanic men and in African-American females [5]. There are estimated to be 4.1 million Americans aged 12 years or over who abused or were dependent on cannabis, accounting for around $60 \%$ of the illicit drug use disorders overall [6].

The scientific evidence on the nature and treatment of cannabis use disorder, however, has only developed over the past decade. While there is a small but growing litera- 
ture of the efficacy of interventions for cannabis use disorder, there are few reports on the effectiveness of cannabis treatment as it is provided within publicly-funded treatment agencies. While dependence is the most common harm associated with cannabis use, there is also a clear relationship between substance use and crime [7]. As a result there is a growing concern to have a more effective response to drug-related crime than imprisonment and other solely legal justice responses [8]. In the US and Australia, and increasingly across the developing and developed world, drug courts have been introduced to divert drug-using offenders away from prison into programs involving drug-testing, treatment, supervision and courtmandated sanctions for non-compliance $[9,10]$. The ethical issues associated with legally coerced treatment are an important factor in the formation of public policy [11].

In general, coercion has been shown to be a predictor of successful treatment outcome $[12,13]$. Research into the effectiveness of coerced substance use treatment has been conducted over the past three decades and has yielded an inconclusive pattern of results [7]. Some studies have suggested that legally coerced and non-coerced treatment clients do not differ in treatment outcome [14] while other studies have reported that non-coerced clients have superior outcomes [15].

A recent study on aspects of legal coercion and motivation found that legal coercion was positively associated with readiness to change [16]. A UK study using routine treatment monitoring data, however, has reported that legal coercion was associated with higher levels of treatment drop-out [17]. The contrariety of findings on this important social and legal question highlights the need for further examination of the impact of legal coercion on illicit drug treatment outcome. There have been no studies on correlates and outcomes of legal coercion that have focused on the treatment of the most common illicit drug, cannabis.

This study aims to examine the characteristics, treatment completion and 90-day treatment follow-up outcomes of legally coerced versus non-coerced clients presenting to a large, publicly-funded treatment system in Texas, USA from 2000-2005 with cannabis as their principal drug of concern.

\section{Methods}

This study examined a total of 27,198 unduplicated administrative records on adult Texans with cannabis as the primary problem who were treated in federal and state-funded programs between January 1, 2000 and December 31, 2005. Another 217 records of clients who were readmitted during this time period were deleted from the dataset, with only their first admission included in the analyses. These clients represented $11 \%$ of all alcohol and drug clients who entered treatment during this time period $(32 \%$ of all clients had a primary problem with alcohol, $21 \%$ with crack cocaine; and $11 \%$ with heroin).

The dataset was extracted from the Behavioral Health Integrated Provider System (BHIPS), which is an Internetbased system of administrative records developed by the Texas Department of State Health Services (DSHS), formerly the Texas Commission on Alcohol and Drug Abuse. BHIPS provides record keeping, data sharing within a service network, and supports state and federal administrative data reporting requirements, including the federal Treatment Episode Data System (TEDS). Reimbursement for services is tied to submission of the required client data forms.

Data collected at admission by the treatment programs primarily reflects the living and economic status of the client at that time, as well as substance use of the client before admission, and the number of days in the last month that the client experienced any of the six domains of the Addiction Severity Index (ASI) [18]. Reports are also collected on the client's condition at discharge and at follow-up 90 days after the last service. DSHS requires that follow-up contact be made in person or by telephone. If the client cannot be located, information about the client may be obtained from family members, case worker, parole or probation officers, or other persons (provided the client had given written permission to make such contacts).

BHIPS also collects information from the treatment programs on Diagnostic and Statistical Manual of Mental Disorders IV (DSM-IV), although there are inconsistencies in the extent of reporting the diagnoses since programs without staff trained to do the DSM diagnosis do not report those data.

\section{Analyses}

Means are reported for continuous data and categorical variables are described in percentages. When comparisons between non-coerced and coerced clients are made, t-tests are used for comparisons between normally distributed continuous data and $\chi^{2}$ for categorical data. Binominal odds ratios were calculated using SAS v9 PROC GENMOD, which can model categorical, ordinal, and continuous responses. Variables that approached a significance of $p<.10$ were included in logistic regression analyses to identify independent predictors of being coerced to treatment, completing treatment, and being abstinent at 90day follow-up. Because clients within a local program might have characteristics more similar to each other than those randomly selected from other programs, the Gener- 
alized Estimating Equation (GEE) model was used to account for the variation in user characteristics due to treatment programs. Significance was set at $p<0.05$ using the GEE parameter estimates. No research on humans was carried out by the authors. The secondary analysis of this administrative dataset was approved by the Institutional Review Board of the University of Texas at Austin.

\section{Results \\ Characteristics of Non-coerced and Coerced Clients at Admission}

Texas treatment programs which are funded by DSHS are public community mental health centers or non-profit private entities and represent approximately $72 \%$ of treatment services in Texas. They are licensed to meet health and safety standards and are contracted to provide specific treatments which are reimbursed based on units of service. Eligibility is based on clinical and financial need. Thus, individuals with the means to enter private treatment are not included in this dataset.

Some $69 \%$ of cannabis admissions were involved with the criminal justice system, including those who had a legal status (awaiting trial, diverted to treatment, on probation, parole, or in jail) and those referred to treatment from a criminal justice source (probation, parole, police, or courts). Referral sources for non-coerced admissions included self $(20 \%)$, social services or protective services $(26 \%)$, community mental health centers $(8 \%)$, family or friends $(6 \%)$, or local councils on alcohol and drugs (6\%).

Some $47 \%$ of the clients entered programs which reported DSM-IV diagnoses, and of those diagnosed, $84 \%$ of the coerced and $69 \%$ of the voluntary clients had no condition on Axis I or Axis II. However, some 7\% of the coerced and $14 \%$ of the voluntary clients had a mood depressive disorder $\left(\chi^{2} 284.5, p<.0001\right)$ and another $4 \%$ of coerced and $9 \%$ of voluntary clients were diagnosed with bipolar disorders $\left(\chi^{2} 283.7, p<.0001\right)$. At admission, $6 \%$ of allcoerced and $13 \%$ of all non-coerced patients were prescribed anti-depressant or anti-anxiety medications $\left(\chi^{2}\right.$ $400.70, p<.0001)$ with less than $2 \%$ of clients being prescribed any other medication at admission.

Of those patients who received a substance-related DSMIV diagnosis, 24\% were diagnosed as cannabis abusers, $55 \%$ were cannabis dependent, and 9\% were polysubstance dependent. Fifty-five percent of the coerced clients and $54 \%$ of the voluntary clients met the criteria for cannabis dependence ( $p=0.1098), 26 \%$ of the coerced and $18 \%$ of the non-coerced met the criteria for cannabis abuse $(p<.0001)$, and $8 \%$ of the coerced and $12 \%$ of the non-coerced met the criteria for polysubstance dependence $(p<.0001)$,
These voluntary or non-coerced referrals, as compared to coerced clients, entered treatment with more days of problems in the past month as measured on the six domains of the ASI, they were more likely to be homeless, to have sought care for themselves at hospitals or emergency rooms at least once in the past year, to have used cannabis daily in the 6 months prior to admission, and to have been placed on medication for depression or anxiety problems at admission (Table 1).

To determine which demographic and psych-social functioning characteristics best predict criminal justice status, binominal and multinominal logistic regression models were constructed using referral status $(1=$ coerced and $0=$ non-coerced admission). As shown in Table 1, being male, employed at admission, having health problems not related to substance use, and being younger predicted being coerced into treatment, while being homeless, seeking care in the emergency room or hospital at least once in the past year, using daily, and having more days of family, psychological, or alcohol or drug problems in the month prior to admission as measured on the ASI predicted being a voluntary or non-coerced client.

Some $75 \%$ of the clients who entered treatment with a primary problem with cannabis received outpatient services, with 20\% receiving residential services and 3\% receiving detoxification services. Voluntary clients were more likely to receive detoxification (5\% versus $2 \%, \chi^{2} 166.7, p<$ .0001 ) and residential services (36\% versus $18 \%, \chi^{2}$ 979.9, $p<.0001)$, and less likely to be in outpatient care (64\% versus $\left.80 \%, \chi^{2} 862.8, p<.0001\right)$.

\section{Characteristics of Non-coerced and Coerced Clients Who Completed Treatment}

At discharge, $34 \%$ of non-coerced and $42 \%$ of coerced patients successfully completed the treatment service for which they were enrolled and they were either discharged or referred to an additional level of treatment at another location $\left(\chi^{2} 136.56, p<.0001\right)$. Another $17 \%$ of noncoerced and $21 \%$ of coerced were discharged because of violation of program rules $\left(\chi^{2} 48.50, p<.0001\right)$ and $12 \%$ of non-coerced and $6 \%$ of coerced left against program advice $\left(\chi^{2} 302.9, p<.0001\right)$. Seventy-five percent of the non-coerced and $71 \%$ of the coerced clients were abstinent in the last month of their treatment before discharge $\left(\chi^{2} 31.05, p<.0001\right)$.

The average length of stay from admission to date of the last face-to-face treatment contact was 70 days for coerced clients, as compared to 57 days for non-coerced clients ( $p$ $<.0001)$. Coerced clients stayed in residential longer (31 days versus 28 days, $p<.0001$ ) and in outpatient longer (78 days versus 73 days, $p<.0001$ ). 
Table I: Characteristics of Clients at Admission to Treatment with a Primary Problem with Cannabis. Based on Their Legal Status and Odds Ratios Predicting Legally Coerced Status at Admission: 2000-2005

\begin{tabular}{|c|c|c|c|c|c|c|c|}
\hline \multirow[b]{3}{*}{ Risk Factor } & \multirow{3}{*}{$\begin{array}{l}\text { Voluntary } \\
n=8422\end{array}$} & \multirow{3}{*}{$\begin{array}{l}\text { Coerced } \\
n=18776\end{array}$} & \multirow[b]{3}{*}{$P$ value } & \multicolumn{4}{|c|}{ Multivariate } \\
\hline & & & & \multirow[b]{2}{*}{$\begin{array}{c}\text { Odds Ratios } \\
n=24622\end{array}$} & \multirow[b]{2}{*}{$\operatorname{Pr}>Z$} & \multicolumn{2}{|c|}{$95 \% \mathrm{Cl}$} \\
\hline & & & & & & Lower & Upper \\
\hline Average Age & 28.4 & 26.4 & **** & 0.97 & $* * *$ & 0.96 & 0.98 \\
\hline Male & $46.8 \%$ & $72.2 \%$ & $* * *$ & 2.35 & $* * *$ & 2.04 & 2.71 \\
\hline Black & $25.9 \%$ & $28.7 \%$ & $* * *$ & 1.10 & & 0.71 & 1.71 \\
\hline White & $48.2 \%$ & $39.8 \%$ & $* * *$ & 1.00 & & & \\
\hline Hispanic & $24.1 \%$ & $29.8 \%$ & $* * *$ & 0.96 & & 0.61 & 1.52 \\
\hline Employed at Admission & $22.2 \%$ & $45.4 \%$ & $* * *$ & 2.13 & $* * *$ & 1.84 & 2.45 \\
\hline Placed on Medication at Admission & $12.8 \%$ & $5.7 \%$ & $* * *$ & 0.87 & & 0.71 & 1.05 \\
\hline I or More Past Year ER/Hospital Visits & $39.8 \%$ & $23.2 \%$ & **** & 0.92 & $* * *$ & 0.88 & 0.95 \\
\hline Homeless & $8.5 \%$ & $1.6 \%$ & $* * *$ & 0.28 & $* * *$ & 0.21 & 0.37 \\
\hline Days of Health Problems & 4.3 & 2.6 & $* * *$ & 1.01 & $* * *$ & 1.00 & 1.01 \\
\hline Days of Employment Problems & 11.3 & 7.3 & *** & 1.00 & & 0.99 & 1.01 \\
\hline Days of Family Problems & 10.2 & 4.8 & *** & 0.98 & $* * *$ & 0.98 & 0.99 \\
\hline Days of Social Problems & 6.8 & 3.4 & $* * *$ & 1.00 & & 0.99 & 1.01 \\
\hline Days of Psychological Problems & 10.7 & 5.0 & $* * *$ & 0.98 & $* * *$ & 0.98 & 0.99 \\
\hline Days of Drug/Alcohol Problems & 11.6 & 6.7 & $* * *$ & 0.99 & $* *$ & 0.99 & 1.00 \\
\hline Used Daily in Last 6 Months & $47.4 \%$ & $33.0 \%$ & $* * *$ & 0.68 & $* * *$ & 0.61 & 0.76 \\
\hline
\end{tabular}

$*_{\mathrm{p}}=<.05$,

$*^{* *} \mathrm{p}=<.01$

$* * * \mathrm{p}=<.0001$

Since coerced clients were more likely to complete treatment, data were analyzed to determine which variables predicted completing treatment. A binominal model was constructed using the six ASI variables, length of stay in treatment, number of family and friends involved in the treatment process, abstinence in the last month of treatment, being in residential treatment, and number of 12 Step meetings attended in the last month of treatment. Those variables which were significant were then included in multinominal models for coerced and non-coerced clients $(1=$ completed treatment and $0=$ non-completer $)$. As Table 2 shows, for both groups, being abstinent in the month prior to discharge, having more family and friends involved during treatment, attending more 12-step meet- ings during the last month of treatment, and a longer length of stay were significant factors in predicting treatment completion, while having more days of psychological problems in the month before admission predicted non-completion.

\section{Status of Clients at 90 day Follow-up}

Information on status of clients 90 days after last treatment encounter was obtained on $68 \%$ of the clients. Contact was more likely to be made with coerced clients $(75 \%$ versus $\left.66 \%, \chi^{2} 179.6, p<.0001\right)$. Of the clients, $84 \%$ of coerced and $77 \%$ of the non-coerced clients had not used cannabis in the month prior to follow-up $\left(\chi^{2} 77.2, p<\right.$ $.0001)$.

Table 2: Multivariate Prediction of Treatment Completion for Legally Coerced and Voluntary Clients with a Primary Problem with Cannabis: 2000-2005

\begin{tabular}{|c|c|c|c|c|c|c|c|c|}
\hline \multirow[b]{3}{*}{ Risk Factor } & \multicolumn{4}{|c|}{$\begin{array}{l}\text { Criminal Justice Admissions } \\
\qquad n=13822\end{array}$} & \multicolumn{4}{|c|}{$\begin{array}{l}\text { Voluntary Admissions } \\
\quad n=5799\end{array}$} \\
\hline & \multirow[b]{2}{*}{ Odds Ratios } & \multirow[b]{2}{*}{$\operatorname{Pr}>Z$} & \multicolumn{2}{|c|}{$95 \% \mathrm{Cl}$} & \multirow[b]{2}{*}{ Odds Ratios } & \multirow[b]{2}{*}{$\operatorname{Pr}>\mathrm{Z}$} & \multicolumn{2}{|c|}{$95 \% \mathrm{Cl}$} \\
\hline & & & Lower & Upper & & & Lower & Upper \\
\hline Days of Psychological Problems at Admission & 0.99 & **** & 0.98 & 0.99 & 0.99 & *** & 0.98 & 0.99 \\
\hline Length of Stay & 1.01 & $* * *$ & 1.00 & 1.01 & 1.01 & *** & 1.01 & 1.01 \\
\hline Family \& Friends Involved in Treatment Process & 1.18 & $* * *$ & 1.10 & 1.27 & 1.13 & $* *$ & 1.06 & 1.20 \\
\hline Abstinent Last 30 Days of Treatment & 7.95 & $* * *$ & 6.58 & 9.61 & 5.57 & $* * *$ & 4.41 & 6.78 \\
\hline $\begin{array}{l}\text { 12-Step Meetings Attended in Last } 30 \text { Days of } \\
\text { Treatment }\end{array}$ & 1.09 & $* * *$ & 1.07 & I.II & 1.07 & *** & 1.06 & 1.08 \\
\hline
\end{tabular}

$*_{p}=<.05$

*** $=<.01$

$* * * \mathrm{p}=<.0001$ 
Binominal models were constructed to predict pastmonth abstinence at follow-up ( $1=$ no use and $0=$ use $)$ using number of months employed since discharge, whether or not the client was living in a household where he or she was exposed to alcohol abuse or drug use, number of arrests since discharge, number of emergency room or hospital visits since discharge, all the ASI problem indices except days of alcohol or drug problems, number of 12-Step meetings attended in the month prior to follow-up, and whether or not the client had been in residential treatment. Those variables which were significant were then included in multinominal models for coerced and non-coerced clients (Table 3 ).

For both groups, living in situations where they were exposed to alcohol abuse or drug use and having more days of employment, family, or psychological problems in the month prior to follow-up predicted not being abstinent at follow-up, while attending more 12-Step meetings in the previous month predicted past-month abstinence for both groups. For coerced clients, cannabis use at follow-up was also predicted by more arrests since discharge, while for voluntary clients, having been in residential treatment and having more days of social problems in the month before follow-up predicted cannabis use.

Overall, clients admitted to residential services were more impaired than those admitted to outpatient services, yet they were more likely to be abstinent in their last month of treatment ( $87 \%$ versus $67 \%, \chi^{2} 870.2, p<.0001$ ) and to complete treatment $\left(70 \%\right.$ versus $53 \%, \chi^{2} 578.4, p<$ $.0001)$. But they were less likely to be abstinent at followup $\left(34 \%\right.$ versus $\left.44 \%, \chi^{2} 207.8, p<.0001\right)$.

\section{Discussion}

This study has found that individuals in state-funded alcohol and other drug services in Texas who were coerced into treatment $(69 \%)$ were less impaired and more likely to have completed treatment $(42 \%)$, and be abstinent from cannabis at follow-up (84\%) than their non-coerced peers.

At admission, clients who voluntarily entered treatment were less likely to be employed, to have sought care in an emergency room or hospital in the past year, to have used cannabis daily in the six months prior to admission, and to report more days of family, psychological, and drug and alcohol problems

This study found that $55 \%$ of all the clients met the criteria for cannabis dependence, a number close to the $60 \%$ findings of an Australian study of individuals diverted by police after arrest for cannabis-related offences [19]. Legally coerced clients were less impaired at admission, were more likely to stay in treatment longer and to complete treatment, and were less likely to leave against program advice but were more likely to be asked to leave as a result of rule violations. These findings are in contrast to a recent UK study that reported coerced clients were more likely to drop out of treatment [17]. Participants in that study who were legally coerced into treatment were also more likely to receive less intensive forms of treatment (outpatient counseling), to be contacted at follow-up, and to have not used in the month prior to that contact. The authors concluded that their coerced status contributed to their longer length of stay so that they were more likely to have successfully completed outpatient treatment. The differences in the study outcomes may be accounted for by differences in illicit drug use. The UK study does not report by principal drug of concern but appears to focus on injecting drug users [17]. These findings highlight the importance of assessing treatment outcome by principal drug of concern.

Table 3: Multivariate Prediction of Past Month Abstinence from Cannabis at 90 Day Follow-Up for Legally Coerced and Voluntary Clients: 2000-2005

\begin{tabular}{|c|c|c|c|c|c|c|c|c|}
\hline \multirow[b]{3}{*}{ Risk Factor } & \multicolumn{4}{|c|}{$\begin{array}{l}\text { Criminal Justice Admissions } \\
\qquad \mathrm{n}=7822\end{array}$} & \multicolumn{4}{|c|}{$\begin{array}{l}\text { Voluntary Admissions } \\
\qquad n=2721\end{array}$} \\
\hline & \multirow[b]{2}{*}{ Odds Ratios } & \multirow[b]{2}{*}{ Pr $>\mathbf{Z}$} & \multicolumn{2}{|c|}{$95 \% \mathrm{Cl}$} & \multirow[b]{2}{*}{ Odds Ratios } & \multirow[b]{2}{*}{ Pr $>\mathbf{Z}$} & \multicolumn{2}{|c|}{$95 \% \mathrm{Cl}$} \\
\hline & & & Lower & Upper & & & Lower & Upper \\
\hline $\begin{array}{l}\text { Living in Household Where Exposed to Alcohol } \\
\text { Abuse or Drug Use }\end{array}$ & 0.15 & $* * *$ & 0.11 & 0.21 & 0.10 & $* * *$ & 0.07 & 0.15 \\
\hline Arrests Since Discharge & 0.93 & $*$ & 0.86 & 1.00 & 0.75 & & 0.48 & 1.15 \\
\hline Days of Employment Problems at Follow-up & 0.97 & $* * *$ & 0.96 & 0.98 & 0.97 & $* *$ & 0.95 & 0.99 \\
\hline Days of Family Problems at Follow-Up & 0.96 & $* * *$ & 0.95 & 0.98 & 0.97 & $* *$ & 0.95 & 0.99 \\
\hline Days of Social Problems at Follow-Up & 0.99 & & 0.97 & 1.03 & 0.95 & $* * *$ & 0.94 & 0.97 \\
\hline Days of Psychological Problems at Follow-Up & 0.95 & $* * *$ & 0.94 & 0.96 & 0.97 & $* *$ & 0.95 & 0.99 \\
\hline 12-Step Meetings Attended in Last 30 Days & 1.13 & $* * *$ & 1.09 & 1.17 & 1.08 & $* *$ & 1.04 & 1.13 \\
\hline Residential Treatment & 0.86 & & 0.63 & 1.16 & 0.58 & $* *$ & 0.44 & 0.78 \\
\hline
\end{tabular}

\footnotetext{
$*_{p}=<.05$

$* * \mathrm{p}=<.01$

$* * * \mathrm{p}=<.0001$
} 
Having more days of psychological problems was a predictor of voluntary status at treatment admission, a predictor of non-completion of treatment, and a predictor of cannabis use at follow-up, regardless of legal status. Although the level of co-occurring disorders may be underreported in this dataset, the diagnoses of depression and anxiety, particularly among the voluntary clients, and the prescribing of medications for these conditions provides evidence that treatment of co-occurring conditions would be a factor in increasing positive treatment outcomes.

Family and friends affected treatment outcomes both positively and negatively. Having more days of family problems at admission was a predictor of voluntary status and it was a predictor of cannabis use at follow-up for both non-coerced and coerced clients. Individuals who had more family and friends involved in their treatment process were more likely to complete treatment, while, as expected, living in a household where the individuals were exposed to alcohol abuse or drug use predicted cannabis use at follow-up. The finding that having been in a residential program was associated with abstinence in the last month of treatment, completing treatment, but not being abstinent at follow-up may be related to the benefits of a more structured setting in treatment and the lack of a stable and sober living environment after treatment.

Employment was also an important factor in this study. At admission, coerced clients were more likely to be employed and to report fewer days of employment problems as measured on the ASI employment domain. At follow-up, both coerced and voluntary clients who reported more days of employment problems were more likely to be using cannabis. Likewise, more attendance at 12-step meetings was a significant predictor of treatment completion and abstinence from cannabis in the month preceding treatment discharge and in the month preceding 90day follow-up.

\section{Conclusion}

This report profiles two types of clients who enter treatment in Texas with a primary problem with cannabis: (1) those who are less impaired who were sent to treatment as a result of their involvement in the criminal justice system and (2) those who were more impaired who entered treatment voluntarily. These findings support the positive treatment outcomes for clients legally coerced into cannabis treatment compared to non-coerced clients. The study also highlights the need for a greater range of interventions to assist those with cannabis use disorders before they develop the range and severity of health and psychosocial problems seen among these non-coerced clients. Early $[20,21]$ and brief $[22,23]$ interventions have been shown to be effective in the management of cannabis use disorders. The raising of public awareness of the prevalence of cannabis use disorders and the availability and efficacy of subsidised, earlier stage and briefer interventions would reduce the burden of these disorders. Screening, detecting and intervening in the early stages of cannabis abuse would reduce the need for more intensive residential services for severely impaired cannabisdependent individuals.

Given the high rates of physical and psychological health problems and frequency of contact with the emergency rooms, there is an urgent need for routine screening for cannabis use disorders in primary health care settings. Training clinicians in the delivery of these 1-2 session interventions comprising motivational enhancement and cognitive behavioural therapy skills, in a variety of settings including adolescent health services, juvenile justice facilities and primary health care, would further improve cannabis treatment outcomes at earlier stages of the disorder. Those clients with severe psycho-social and health problems associated with their cannabis use should have greater access to subsidised specialist residential treatment services that have expertise in the management of comorbid psychiatric and cannabis use disorders.

While this is a large dataset, it is representative only of lower income clients who entered publicly-funded treatment in Texas. The 90-day follow-up data is largely selfreported and was not biochemically validated and the reporting on DSM-IV diagnoses was not uniform across all programs. The size of the dataset and the large number of variables in BHIPS, however, provides insight into treatment characteristics and the short-term treatment outcomes of those individuals who develop problems due to their cannabis use. This study did not analyze treatment outcome by type of legal coercion employed. Coercion is a complex psychological construct and this study was not able to assess issues such as resistance to treatment, which has been shown to be an important factor in treatment outcome, particularly among those coerced into treatment [24]. Future research should address these limitations.

\section{Competing interests}

The author(s) declare that they have no competing interests.

\section{Authors' contributions}

JC guided aspects of the analyses and was the lead author on the introduction and conclusions sections of the paper. JCM secured access to the data, performed the analyses and was the lead author of the methods, results, and discussion sections. Both authors read and approved the final manuscript. 


\section{Acknowledgements}

The authors would like to thank the Texas Department of State Health Services for the use of their client data.

\section{References}

I. United Nations: Social and Economic Costs of Illicit Drugs New York: United Nations; 1998.

2. United Nations Office of Drugs and Crime: World Drug Report Volume I. Vienna, UNODC; 2006.

3. Chen C-Y, O'Brien MS, Anthony JC: Who becomes cannabis dependent soon after onset of use? Epidemiological evidence from the United States: 2000-200I. Drug and Alcohol Dependence 2005, 79(I): I I-22.

4. Grant BF, Pickering R: The relationship between cannabis use and DSM-IV cannabis abuse and dependence: results from the National Longitudinal Alcohol Epidemiologic Survey. Journal of Substance Abuse 1998, 1 0:255-264.

5. Compton WM, Grant BF, Colliver JD, Glantz MD, Stinson FS: Prevalence of marijuana use disorders in the United States: I99 I1992 and 2001-2002. Journal of the American Medical Association 2004, 29I:2I|4-2I 2 I.

6. Substance Abuse and Mental Health Services Administration: Results from the 2005 National Survey on Drug Use and Health: National Findings. In Office of Applied Studies, NSDUH Series $\mathrm{H}-30$, DHHS Publication No. SMA 06-4 194. Rockville, MD ; 2006.

7. Klag S, O'Callaghan F, Creed $P$ : The use of legal coercion in the treatment of substance abusers: an overview and critical analysis of thirty years of research. Substance Use \& Misuse 2005 40:1777-1795

8. Bull M: A comparative review of best practice guidelines for the diversion of drug related offenders. International Journal of Drug Policy 2005, 16:223-234.

9. Makkai T: The emergence of drug treatment courts in Australia. Substance Use \& Misuse 2002, 37:1567-1594.

10. Office of National Drug Control Policy: National Drug Control Strategy. Washington: The White House; 2005.

II. Caplan AL: Ethical issues surrounding forced, mandated, or coerced treatment. Journal of Substance Abuse Treatment 2006, $31: 117-120$

12. Ciraulo D, Piechniczek-Buczek J, Iscan E: Outcome predictors in substance use disorders. Psychiatric Clinics of North America 2003, 26:38I-409.

13. Miller N, Flaherty J: Effectiveness of coerced addiction treatment (alternative consequences). A review of the clinical research. Journal of Substance Abuse Treatment 2000, 18:9-16.

14. Farabee D, Prendergast M, Anglin MD: The effectiveness of coerced treatment for drug abusing offenders. Federal Probation 1998, 62:3-10

15. Simpson D, Joe G, Rowan-Szal G: Drug abuse treatment retention and process effects on follow-up outcomes. Drug and Alcohol Dependence 1997, 47:227-235.

16. Gregoire T, Burke A: The relationship between legal coercion to readiness to change among adults with alcohol and other drug problems. Journal of Substance Abuse Treatment 2004, 26(I):337-343.

17. Beynon CM, Bellis MA, McVeigh J: Trends in drop out, drug free discharge and rates of re-presentation: a retrospective cohort study of drug treatment clients in the North West of England. BMC Public Health 2004, 6:205.

18. McLellan A, Luborsky L, Woody G, O'Brien C: An improved evaluation instrument for substance abuse patients: the addiction severity index. Journal of Nervous and Mental Disease 1980, I 68(I):26-33.

19. Feeney G, Connor J, Young R, Tucker J, McPherson A: Cannabis dependence and mental health perception amongst people diverted by police after arrest for cannabis-related offending behavior in Australia. Criminal Behavior and Mental Health 2005, I 5(4):249-260.

20. Martin G, Copeland J, Swift W: Adolescent Cannabis Check-up: feasibility study. Journal of Substance Abuse Treatment 2005, 29:207-2I3.

21. McCambridge J, Strang J: The efficacy of single-session motivational interviewing in reducing drug consumption and perceptions of drug-related risk and harm among young people: results from a multi-site cluster randomized trial. Addiction 2004, 99:39-52.

22. Copeland J, Swift W, Roffman R, Stephens R: A randomised controlled trial of brief interventions for cannabis use disorder. Journal of Substance Abuse Treatment 200I, 2 I:55-64.

23. Marijuana Treatment Project Group: Brief treatments for cannabis dependence: findings from a randomized controlled trial. Journal of Consulting and Clinical Psychology 2004, 72:455-466.

24. Longshore $D$, Teruya $C$ : Treatment motivation in drug users: a theory-based analysis. Drug and Alcohol Dependence 2006, 8 I: $179-188$

\section{Pre-publication history}

The pre-publication history for this paper can be accessed here:

\section{http://www.biomedcentral.com/1471-2458/7/111/pre}

pub
Publish with Biomed Central and every scientist can read your work free of charge

"BioMed Central will be the most significant development for disseminating the results of biomedical research in our lifetime. "

Sir Paul Nurse, Cancer Research UK

Your research papers will be:

- available free of charge to the entire biomedical community

- peer reviewed and published immediately upon acceptance

- cited in PubMed and archived on PubMed Central

- yours - you keep the copyright 